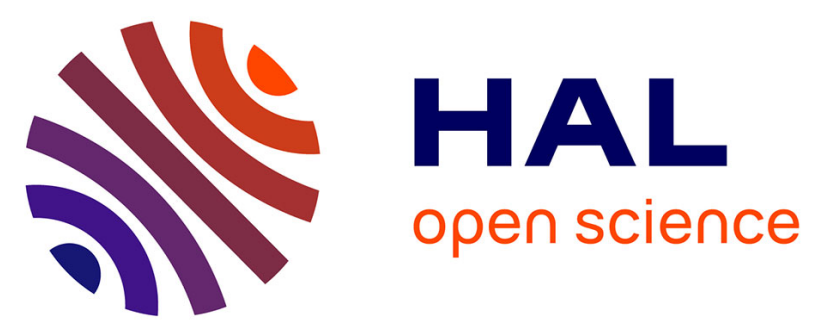

\title{
La sidérurgie dans l'est de la Gaule: l'organisation spatiale de la production de l'Âge du Fer au haut Moyen Âge
}

Marc Leroy, Michel Mangin, Hervé Laurent, Mostefa Boukezzoula, Baraka Raïssouni

\section{To cite this version:}

Marc Leroy, Michel Mangin, Hervé Laurent, Mostefa Boukezzoula, Baraka Raïssouni. La sidérurgie dans l'est de la Gaule: l'organisation spatiale de la production de l'Âge du Fer au haut Moyen Âge. Gallia - Archéologie de la France antique, 2000, Mines et métallurgies en Gaule, 57, pp.11-21. 10.3406/galia.2000.3206 . hal-01902757

\section{HAL Id: hal-01902757 https://hal.science/hal-01902757}

Submitted on 20 Jan 2020

HAL is a multi-disciplinary open access archive for the deposit and dissemination of scientific research documents, whether they are published or not. The documents may come from teaching and research institutions in France or abroad, or from public or private research centers.
L'archive ouverte pluridisciplinaire HAL, est destinée au dépôt et à la diffusion de documents scientifiques de niveau recherche, publiés ou non, émanant des établissements d'enseignement et de recherche français ou étrangers, des laboratoires publics ou privés.

\section{(ㅇ)(1) $\$$}

Distributed under a Creative Commons Attribution - NonCommercial - NoDerivatives $\mid 4.0$ 


\title{
LA SIDÉRURGIE DANS L'EST DE LA GAULE
}

\author{
L'organisation spatiale de la production \\ de l'âge du Fer au haut Moyen Âge
}

\author{
Marc Leroy, Michel Mangin, Hervé Laurent, \\ Mostefa BoukEzzoula et Baraka RAÏssounI
}

Mots-clés. Sidérurgie ancienne, Gaule de l'Est, réduction, forge, districts de production.

Key-words. Early ironworking, Eastern France, bloomeries, smithing workshops, production area.

\begin{abstract}
Résumé. Dans l'est de la France, des programmes de recherche mis en æuvre à partir du milieu des années 1980 ont abouti à la cartographie de plus de 1000 sites miniers et sidérurgiques anciens, répartis inégalement sur les trois régions administratives de Bourgogne, Lorraine et Franche-Comté. Il s'agit du plus vaste espace géographique en Furope occidentale sur lequel a été développée une problématique spécifique associant étroitement deux aspects complémentaires : d'une part, l'établissement d'une carte des sites d'extraction minière et de transformation métallurgiques antérieurs au développement du haut fourneau; d'autre part, le traitement archéométrique des données recueillies sur le terrain, pour définir la nature et la place des sites dans le conlexte géographique et historique de chaque période étudiée. Les données acquises permettent désormais de brosser un premier bilan historique de l'organisation et de l'évolution de la sidérurgie ancienne dans l'est de la Gaule, au moins depuis la fin de l'âge du Fer jusqu'au haut Moyen Âge.
\end{abstract}

Abstract. In the East of France, since the beginning of the 80's, recent researches in the regions of Bourgogne, Franche-Comte and Lorraine have provided archaeological data of more than 1000 early ironworking sites (mining, smelting and smithing places). It is the largest area in Western Europe where this kind of research including both archaeological survey and archaeometrical investigations was developed to determine the place of each site in the metallurgical process. So several production areas have been delimited. Now, it is possible to present the first schemes of organization of early ironworking in Eastern Gaul and its evolution, from the end of Iron Age to Early Middle Ages.

$\mathrm{Si}$, depuis les premières recherches archéologiques dans le courant du XIX ${ }^{e}$ s., on savait que l'Occident continental européen au nord des Pyrénées était riche en vestiges de travail du fer selon le procédé direct, aucune carte archéométallurgique régionale n'avait encore été établie en France, au début des années 1980. La mise en route de programmes d'inventaire systématique, en Bourgogne à partir de 1981, en Franche-Comté à partir de 1983 et en Lorraine à partir de 1988, a permis d'aboutir, en un peu plus d'une dizaine d'années, à la réalisation d'une carte qui comporte près d'un millier de sites miniers et métallúrgiques répartis très inégalement sur douze départements de ces trois régions administratives ${ }^{6}$. Elle reste, à ce jour, la plus importante cartographie réalisée en France par l'espace concerné et le nombre de sites

6. Une première synthèse a été présentée lors du Symposium international du Comité pour la sidérurgie ancienne de l'UISPP, tenu à Sevenans (Territoire de Belfort) en 1990, et publiée dans les actes (Faivre et al., 1995). Une carte réactualisée est présentée dans Mangin éd., 1994. 
répertoriés ${ }^{7}$, mais d'autres espaces géographiques, dans le Centre, l'Ouest et le Sud-Ouest, présentent depuis peu des bilans importants, encore largement inédits.

\section{PROSPECTIONS-INVENTAIRES ET ARCHÉOMÉTRIE : \\ LA CARTOGRAPHIE RAISONNÉE DES SITES ARGHÉOMÉTALLURGIQUES}

\section{DES PROGRAMMES THÉMATIQUES RÉGIONAUX}

La problématique générale qui sous-tend l'ensemble de la recherche dans l'est de la France comporte deux aspects complémentaires, étroitement associés dès l'origine :

- l'établissement d'un inventaire archéologique des sites d'extraction minière et de transformation métallurgique antérieurs à l'apparition du haut fourneau ;

- le traitement des données de terrain, d'une part pour définir la nature des activités pratiquées, grâce à l'étude des vestiges collectés, d'autre part pour préciser la place des sites dans le contexte géographique et humain de chaque période historique.

Dans ce cadre général, on tente de définir les caractéristiques propres à chaque espace géographique ou chaque zone de production en l'étudiant avec une problématique adaptée.

En Bourgogne, dans le Morvan du nord (dès 1982 : Mangin et al., 1992) et dans le Mâconnais (entre 1986 et 1990: Boukezzoula, 1995), il s'agissait d'étudier une zone de production dont l'intérêt ne faisait guère de doute, à en juger par les indications fournies par la bibliographie ancienne. Ainsi dans le Morvan, "le but était de délimiter l'extension et de définir l'organisation et le fonctionnement d'un district minier et métallurgique dans une région où l'extraction du minerai au $\mathrm{XIX}^{\mathrm{c}} \mathrm{s}$. avait suscité l'établissement d'un inventaire qui semblait prometteur" (Mangin et al., 1992, p. 13-14). Dans le Haut-Auxois autour d'Alésia (depuis 1987 : Mangin et al., 2000a), il s'agissait de savoir si les quelques sites métallurgiques répertoriés liés à des habitats entraient dans une série notable, de caractériser le type

7. Pour l'historique des recherches dans chacune des trois régions concernées, on se reportera à la partie introductive des Actes du colloque international de palémétallurgie de Besancon, of. Mangin éd., 1994, I, La carte archéométallurgique, p. 11-15. d'activité (réduction ou forge) et de définir les relations pouvant exister entre cette "métallurgie rurale " et l'important centre d'Alésia.

En Franche-Comté, l'objectif primordial était d'établir l'inventaire des sites sidérurgiques à l'échelle d'une région administrative. En l'absence de publications anciennes, on mesurait très mal l'importance de la sidérurgie de réduction directe. Malgré l'impression généralement partagée par les archéologues, qui faisaient remonter la tradition métallurgique locale jusqu'à la Protohistoire, rien ne permettait d'assurer que la région avait connu une production notable avant la fin du Moyen Âge. Il était essentiel également de délimiter les éventuelles zones privilégiées et d'en définir les caractères, par exemple la forêt d'Arne ou le Finage dolois (Boukezzoula et al., 1990 ; Raïssouni, 1995 ; Laurent, 1996).

En Lorraine, c'est une problématique spécifique, relative à l'utilisation d'un type particulier de minerai de fer, qui a été développée. Il s'agissait de définir si le minerai oolithique, ou "minette " (minerai sédimentaire à faible teneur en fer), avait été employé en réduction directe de manière épisodique ou beaucoup plus fréquemment, alors que d'autres minerais plus riches (jugés a priori plus faciles à utiliser en bas fourneaux) étaient aussi assez largement accessibles. Les recherches étaient donc concentrées sur les parties centrale et septentrionale de la région (plateaux des Côtes de Moselle), là où affleurait la " minette »; mais un inventaire des sites était également conduit à l'échelle de la région tout entière pour replacer les secteurs étudiés dans un cadre géographique et historique plus large (Leroy, 1997).

\section{DES APPROCHES ARCHÉOLOGIQUES ET ARCHÉOMÉTRIQUES ÉTROITEMENT IMBRIQUÉES}

Dans l'ensemble des zones d'étude délimitées à l'intérieur de ce vaste espace géographique, les inventaires archéologiques réalisés à partir de dépouillements bibliographiques et d'enquêtes de terrain (prospectionsinventaires pédestres et aériennes) n'auraient pu suffire à en définir les caractères. Ces recherches ont été, d'emblée, liées à un programme d'analyses chimiques et minéralogiques des déchets de production prélevés sur les sites archéologiques (programme ArtémisePaléosidérurgie développé au Centre de recherches 
pétrographiques et géochimiques du CNRS : Ploquin, 1994a) qui permet, en première approche, de déterminer le ou les types de minerais utilisés, puis de qualifier plus précisément la nature des produits échantillonnés (scories, éléments de fourneaux, minerais en cours de réduction, fragments métalliques) et des activités pratiquées (réduction ou postréduction) ${ }^{8}$. Plusieurs fouilles d'ateliers de réduction ou de forge, en Côted'Or et dans l'Yonne (Bourgogne), en Meurthe-etMoselle (Lorraine) et dans le Doubs (Franche-Comté), ainsi que le suivi systématique des découvertes fournies par l'archéologie préventive ont alimenté à une autre échelle les données rassemblées par les programmes de prospcction et permis d'aborder l'étude plus détaillée des différents stades de la production et du fonctionnement des ateliers (Leroy et al., 1990 ; Mangin et al., 1992 ; Laurent et al., 1994 ; Dunikowski, Cabboï, 1995 ; Leroy, 1997).

La gestion globale de toutes les données analytiques provenant des analyses chimiques (environ un millier), des examens pétrographiques et métallographiques, de l'exploitation diagrammatique des résultats, permet de développer une méthodologie propre à résoudre, en partie, les problèmes posés :

- établissement d'une « typologie » chimique et minéralogique des scories et sa mise en correspondance avec une typologie morphologique, rapportée aux types de minerais utilisés et aux époques considérées ;

- définition de la nature de l'activité d'un site sidérurgique : réduction, épuration, forge ;

- examen critique de la filiation minerai-scories de réduction et spécification du rôle des éléments chimiques ;

- confrontations entre les différentes zones étudiées.

De ces éléments découlent des interprétations sur l'organisation et sur l'échelle de la production, sur les circuits de distribution des matières brutes ou élaborées et sur les incidences socio-économiques, voire politiques, qui en résultent. À cet égard, la méthodologie utilisée dans l'est de la France apparaît exemplaire dans sa pratique et dans ses résultats. Des programmes développés depuis dans d'autres régions s'en inspirent assez largement.

8. Le détail des procédures employées est développé d'une manière globale dans la partie introductive des Actes du colloque international de paléométallurgie de Besanson (Fluzin, 1994 ; Ploquin, 1994a), enrichi dans Fluzin, Leclère, 1998 et dans Mangin et al., 2000a.

\section{LA MISE EN ÉVIDENCE DE DISTRICTS DE PRODUCTION}

Sur l'ensemble de cet espace géographique, qui correspond peu ou prou aux territoires des cités antiques des Éduens, des Leuques, des Lingons, des Médiomatriques et des Séquanes, la cartographie de plus d'un millier de sites met en évidence des zones de forte concentration qui apparaissent comme autant de " districts " de production primaire. Ceux-ci se définissent, d'un point de vue spatial, comme la limite extrême d'une large concentration de vestiges d'extraction minière et d'ateliers de réduction (caractérisés au sol par un ou plusieurs amas de scories, qualifiés de "ferriers »), et, d'un point de vue gîtologique, comme un espace possédant des ressources de minerai de fer effectivement exploitées ${ }^{9}$. Ces districts sidérurgiques sont au moins au nombre de quatre (fig. 2) : le Morvan du nord (Mangin et al., 1992), la forêt d'Arne à l'ouest de Besançon/ Vesontio (Laurent, 1996), le Mâconnais (Boukezzoula, 1995), les Côtes de Moselle en Lorraine centrale et septentrionale (Leroy, 1997).

Les connaissances actuellement bien assurées portent sur :

- le nombre de sites de réduction (200 dans le Morvan du nord, 150 en Lorraine centrale et septentrionale, une centaine sur le pourtour de la forêt d'Arne, une cinquantaine dans le Mâconnais) ;

- leur répartition dans l'espace (distribués tout le long des Côtes de Moselle ou sur une section de la Côte mâconnaise, ou bien dispersés sur de petits gisements minéraux dans le Morvan avec deux concentrations seulement) ;

- l'importance quantitative des déchets (depuis des ferriers de plusieurs centaines, voire plusieurs milliers de mètres cubes dans le Morvan ou en Lorraine, jusqu'à de petits amas de quelques dizaines de mètres cubes dans le Morvan, en Lorraine ou en forêt d'Arne) ;

- la période principale d'activité (l'époque romaine pour les sites du Morvan et probablement du Mâconnais ; le haut Moyen Âge en forêt d'Arne ; le haut et le bas Moyen Âge dans la région des Côtes de Moselle);

9. Cf. H. F. Cleere, D. Crossley, The iron industry of the Weald, Leicester University Press, 1985, 395 p. ; G. Magnusson, Bloomery Iron Production in the ('ounty of Jämtland, Sweden, Stockholm, 1986, 437 p. ; Mangin et al., 1992. 


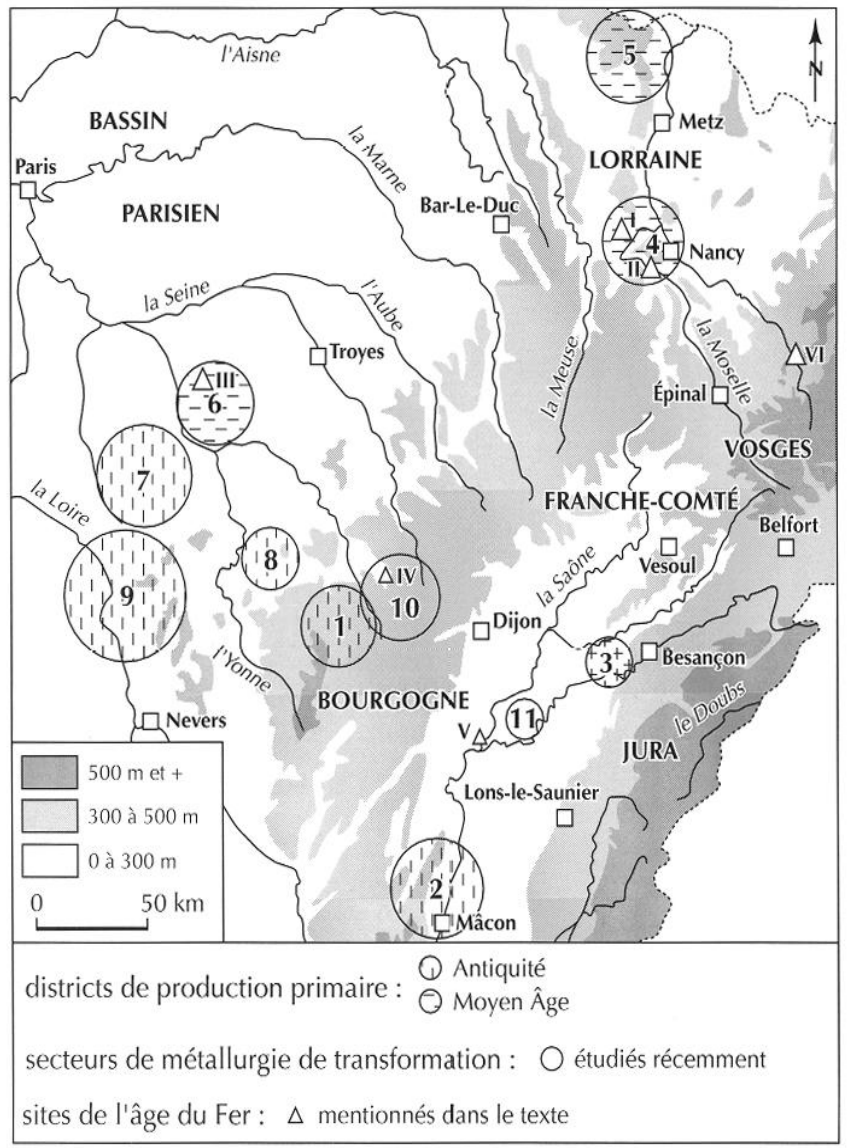

Fig. 2 - Carte de localisation des principaux districts de production sidérurgique identifiés dans l'est de la Caule, pour l'Antiquité el le. Moyen Âge (Ph. Brunella, Service archéologique départemental de la Moselle) : 1, Morvan du nord; 2, Macônnais ; 3, forêt d'Arne; 4, Plaleau de Haye (Côtes de Moselle sud) ; 5, Pays-Haut (Côte de Moselle nord) ; 6, Pays d'Othe; 7, Puisaye; 8 , forêt de Vézelay; 9, Nivernais; 10, Ilaut-Auxois; 11 , Finage dolois ; I, Condreville (Meurthe-et-Moselle) ; II, Camp d'Afjrique (Meurthe-et-Moselle) ; III, Les Clérimois (Yonne) ; IV, Alésia (Côte-d'Or) ; V, Bragnysur-Saône (Saône-el-Loire) ; VI, La Pierre d'Appel, Étival (Vosges).

- les types de fourneaux (petits fourneaux à cheminée dans le Morvan; fourneaux de plus grandes dimensions et très proches morphologiquement en Lorraine et Franche-Comté). Près d'une trentaine d'exemplaires sont actuellement connus dans les limites de l'espace étudié, pour les périodes antérieures au XIII' $s .{ }^{10}$.

10. Il faudrait aussi citer les 18 bas fourncaux fouillés sur le site des Clérimois dans l'Yonne (Dunikowski, Cabboï, 1995), mais l'Yonne, bien que bourguignonne administrativement, est à la fois trop marginale géographiquement et trop importante par sa production pour pouvoir ĉtre intégrée à une présentation de la sidérurgie de l'est de la France.
Bien des questions restent encore plus ou moins largement ouvertes :

- la nature exacte des minerais utilisés et les traces de leur extraction; à cet égard, la Lorraine et le Morvan sont les régions les mieux documentées, respectivement pour les minerais et les vestiges miniers; mais au total, la carte des sites d'exploitation minière de l'Antiquité et du haut Moyen Âge est encore très pauvre et les relations précises entre minerai et atelier restent difficiles à établir ;

- le fonctionnement et le rendement des différents fourneaux ; l'archéométrie a progressé, mais surtout sur des points précis et la synthèse est prématurée : d'une part, les découvertes sont trop récentes et, d'autre part, la diversité des types de fourneaux se révèle bien plus grande que ce qui était admis jusqu'ici ;

- le volume total de la production et sa répartition entre les périodes ne peuvent être encore approchés avec précision, mais des estimations commencent à être proposées pour certains des ateliers les mieux connus (par exemple à Frouard, vallon de Nerbevau : Leroy, 1997) ;

- les courants d'échanges commerciaux ; une fois identifiées les grandes régions de production pour chacune des périodes, on est en droit de penser que les zones qui produisent beaucoup (taille, nombre et répartition des ateliers) exportent vers celles qui produisent moins ou pas du tout; pour apporter des preuves, des pistes de recherche sont ouvertes par l'archéométrie : tracéabilité de certains éléments chimiques propres aux minerais jusque dans les déchets des opérations de postréduction et/ou reliques de certaines morphologies de minerai dans les masses de métal brutes ou semi-élaborées (Serneels, 1994 ; Leroy, 1997 ; Fluzin, Leclère, 1998).

Ces districts, qui figurent parmi les mieux étudiés d'Europe, sont à inscrire dans une cartographie plus générale à l'échelle de la Gaule tout entière, dont une première esquisse vient d'être présentée récemment (Mangin, Serneels, 1996). Ils sont aussi à comparer aux zones productives situées en périphérie immédiate des limites de la zone étudiée : vastes et ayant produit beaucoup, comme en Puisaye, région de Vézelay et forêt d'Othe à l'ouest ; plus limitées dans l'espace sinon dans le temps, comme dans le Jura suisse à l'est, et pour lesquelles des recherches du même ordre sont en cours ( $c f$. Dunikowski, Cabboï, 1995, pour le Sénonais; Serneels, 1993 et Eschenlohr, 1994, pour lc Jura suisse). 


\section{L'ÂGE DU FER : DES DONNÉES ENCORE TRÈS PONCTUELLES}

\section{AVANT LE MILIEU DU SECOND ÂGE DU FER : PEU D'INFORMATION}

Comme partout en Europe, les ateliers sidérurgiques connus dans l'est de la Gaule pour la période antérieure au $\mathrm{III}^{\mathrm{e}} \mathrm{s}$. avant notre ère sont en nombre très limité (Mangin et al., 1992 ; Pleiner, 1994). Des scories ont été récoltées sur une douzaine de sites au plus, la plupart du temps en contexte d'habitat, ouvert ou fortifié. Dans la moitié des cas seulement, les découvertes ont donné lieu à une étude descriptive ou archéométriquc plus poussée. On connaît un seul atelier de réduction assuré avec les restes d'un fourneau très fortement arasé, qui a été fouillé et étudié très récemment (Gondreville, ZAC de la Roseraie, Lorraine, encore inédit) ". Daté de la fin du $V^{\mathrm{e}}$ ou du début du $\mathrm{V}^{\mathrm{c}} \mathrm{s}$. avant notre ère, il est inséré dans un habitat ouvert, à vocation principalement agricole. Grillage et réduction du minerai sont assurés, mais aucun indice d'extraction ou de forgeage n'a été mis en évidence ${ }^{12}$.

Des scories de réduction en petite quantité ont été découvertes sur le site d'habitat ouvert de Bragny-surSaône en Saône-et-loire, $\mathrm{V}^{\mathrm{e}}-\mathrm{IV} \mathrm{V}^{\mathrm{e}} \mathrm{s}$. avant notre ère (Flouest, 1995). Mais l'activité dominante semble concerner les travaux de forgeage. Les vestiges découverts sur les habitats de même époque à Besançon-Saint-Paul (Doubs, Franche-Comté) ${ }^{13}$ et au Camp d'Affrique à Messein (Meurthe-et-Moselle, Lorraine) ${ }^{14}$ se rapportent également à l'activité de mise en forme du métal (et

11. Responsable scientifique : Sylvie Deffressigne, AFAN. Étude paléomëtallurgique (fouille et laboratoire) : Marc Leroy et Paul Merluzio, LAM.

12. Il s'agit d'un mincrai de fer de type oolithique provenant d'une séquence sédimentaire du Bajocien supérieur, mais qui n'est pas de la "minette".

13. P. Pétrequin (dir.), Le gisement néolithique et protohistorique de Besançon/Saint-Paul (Doubs), Annales littéraires de l'université de Besançon, 228, Archćologie, 30, Paris, Les Belles Lettres, 1979.

14. A. Ploquin, J.-P. Lagadec, M. L.eroy, Apports de la pétrographie et de la géochimie à l'interprétation du site protohistorique dit " Camp d'Affrique "(Messein, Meurthe-et-Moselle, Lorraine) : métallurgie, rempart à " noyau calciné ", in: Miskovsky J.-C. (dir.), Les applications de la géologie à la connaissance de l'environnement de l'homme fossile, Mémoires de la Sociétć géologique de France, 160, Paris, Société géologique de France, 1992, p. 55-65. probablement de fabrication d'objets, même si les produits eux-mêmes nous manquent).

La pauvreté des témoins de l'activité sidérurgique pour ces périodes anciennes est à souligner et ce malgré la multiplication, depuis une décennie, des fouilles d'habitat et l'attention portée aux vestiges de travail de l'âge du Fer. Les investigations récentes ne permettent donc pas de savoir si les premiers objets en fer retrouvés dans les sépultures aristocratiques du premier âge du Fer sont des productions locales ou s'il s'agit de biens de prestige faisant l'objet d'échanges commerciaux sur de longues distances entre les élites dominantes. De la même manière, il reste difficile de conforter les hypothèses qui associent l'émergence des pôles princiers au contrôlc des richesses du sous-sol et en particulier à l'exploitation des minerais de fer ${ }^{15}$.

\section{AU SECOND ÂGE DU FER : L'APPARITION DE ZONES DE PRODUCTION SPÉCIALISÉES ?}

Si l'on prend la quantité et la qualité des objets en fer comme seuls critères d'étude, la production semble exploser à la fin de l'âge du Fer (II" et I ${ }^{\text {er }}$ s. avant notre ère), nettement avant l'intervention romaine en Gaule de l'Est (58 avant J.-C.) ${ }^{16}$. Si les oppida et les vici sont nombreux en Bourgogne et en Lorraine, la liste des sites où les forges sont bien connues est encore très brève (une dizaine de sites recensés dans les trois régions administratives concernées, seuls deux d'entre eux ayant fait l'objet d'une étude particulière, fig. 2) ${ }^{17}$. Le volume de fer utilisé dans la construction des nombreux muri gallici du type Avaricum, édifiés avant ou immédiatement après la conquête, est considérable (plusieurs tonnes souvent

15. G. H. Härke, Settlement types and patterns in the West Hallstatt protince, Oxford, BAR International Series, 57, 1979, 298 p.; I.. Olivier, Des chevaux, de l'acier et la puissance. Le passage à l'âge du Fer en Lorraine dans son contexte européen, in: La Lorraine d'avant l'Histoire, du Paléolithique inférieur au premier âge du Fer, musées de Met., Metz, éd. Serpenoise, 1986, p. 148-177; cf. notc 14 : A. Ploquin et al., op. cit., 1992.

16. R. Pleiner, Les débuts du fer en Europe, Dialogues d'histoire ancienne, 8, 1982, p. 167-192.

17. On ne peut en effet guère citer que les ateliers de $\mathrm{L}$ a Pierre d'Appel (Étival, Vosges), cf. A. Deyber, I a paléométallurgie du fer et des métaux non ferreux à l'oppidum de la Pierre d'Appel (Étival, Vosges), mémoire de DEA, univ. d'Aix-Marseille, 1983 et Alésia (Alise-SainteReine, Côte-d'Or), cf. Bénard, 1989 ; Mangin el al., 2000a. 
pour un seul oppidum). Il a toujours paru évident que la production du métal nécessaire avait lieu sur les sites euxmêmes. Cette opinion était renforcée par la présence presque systématique de déchets de travail. Cette idée est encore largement partagée. Or, il s'avère, dans tous les cas où ces déchets ont été étudiés, qu'il s'agit exclusivement de scories de forge (épuration et/ou élaboration d'objets), jamais de scories coulées issues de la réduction. Mieux, aucun atelier de réduction de cette période n'a encore été noté en prospection dans l'environnement proche des oppida et des vici.

Jusqu'à ce que de nouvelles découvertes viennent contredire ce constat, il faut donc admettre que le fer utilisé provenait de l'importation de métal brut ou semiouvré (currency bars, lingots bipyramidaux ou, d'une façon générale, toute forme de "fers marchands"), issu de sites de production spécialisés (Doswald, 1994). Sur l'oppidum du Crêt-Châtelard, dans la Loire, ont été répertoriées 70 tiges de fer qui pourraient constituer un produit préparatoire des fiches du murus gallicus, tiré directement de lingots importés ${ }^{18}$. Il semble que cette question n'ait jamais jusqu'ici été clairement soulevée dans la mesure où, d'une part, on postulait la réduction sur les oppida et où, d'autre part, on ne connaissait aucun site de réduction capable d'approvisionner un tel marché. Or, la découverte d'un site de grande production aux Clérimois dans l'Yonne, fonctionnant dès le $\mathrm{II}^{\mathrm{e}} \mathrm{s}$. avant notre ère, permet maintenant d'envisager cette question sous un jour nouveau ${ }^{19}$. Mais il faudrait dater de la même période d'autres sites de l'est des Gaules. Or, en l'état actuel de la recherche, il ne semble pas qu'il puisse en exister d'équivalents ailleurs que dans l'Yonne. C'est dire qu'il faut poser la question d'un approvisionnement des oppida en lingots provenant - au moins en partie - de quelques centres lointains (plusieurs centaines de kilomètres parfois), ignorant ainsi les frontières de plusieurs peuples. Certains d'entre eux pouvaient-ils admettre de dépendre d'autres peuples - souvent rivaux sinon

18. L. Orengo, La métallurgie antique au Crêt-Chatelard (Loire), mém. de Maîtrise dactylogr., univ. Lyon II, 1995.

19. Parmi les bas fourneaux qui ont produit les $13000 \mathrm{t}$ de scories du site des Clérimois, au nord de Sens, sur le tracé de l'autoroute A5, plusicurs remontent sinon avec certitude au premier âge du Fer, période pour laquelle les datations sont trop peu nombreuses, du moins sans conteste à la fin du $\mathrm{II}^{e}$ s. et au $\mathrm{I}^{\text {er }}$ s. avant notre ère (Dunikowski, Cabboî, 1995). toujours ennemis - pour un produit stratégique de cette importance ? Pourquoi pas? On sait que dès les années 80 avant notre ère, l'intensité des échanges entre les grands peuples du Centre-Est des Gaules avait suscité la formation d'une sorte «d'union monétaire ", la « zone du denier " mise en évidence il y a plusieurs décennies déjà ${ }^{20}$. L'hypothèse d'une importation systématique de grandes quantités de fer produites chez les Sénons (Yonne) ou les Éduens (Morvan) par les autres peuples n'est donc pas absurde. Elle mériterait d'être étudiée d'une façon approfondie ${ }^{21}$.

La production de fer brut dans de grands centres comme Les Clérimois suppose aussi l'existence d'ateliers de première élaboration des lingots qui restent à découvrir: les oppida sur lesquels les « culots» de forge sont abondants et de grande taille en sont-ils des témoins? Il existe, par ailleurs, une corrélation entre la protourbanisation des oppida et des vici et le développement des ateliers de forge ; la métallurgie joue un rôle certain dans cette première urbanisation de type indigène: à Alésia, vers 40 avant J.-C., en bordure de la place publique, les forges alternent avec les ateliers de bronziers dont elles semblent dépendre. Ce système est mis en place par une autorité qui découpe l'espace à urbaniser en lots spécialisés (Bénard, 1989).

\section{LA PÉRIODE ROMAINE : UNE ORGANISATION DE IAA PRODUCTION MIEUX CONNUE}

Alors que le paysage urbain se romanise dès la fin du $\mathrm{I}^{\mathrm{er}} \mathrm{s}$. avant notre ère, la continuité de l'activité sidérurgique est manifeste dans les oppida qui se transforment alors en agglomérations gallo-romaines plus ou moins durables (par exemple Alésia ou Étival). Ce constat est-il valable aussi sur les sites de réduction ? C'est possible, si l'on en juge par les ateliers des Clérimois où les artisans celtes, actifs dès le II' $\mathrm{s}$. avant notre ère, continuent, sur le même site, à produire avec la même technologie pendant tout le Haut-Empire (I ${ }^{\text {er }}-\mathrm{II}^{\mathrm{c}} \mathrm{s}$.) ; mais peut-on généraliser : la production du Morvan du nord semble bien

20. J-B. Colbert de Beaulieu, Traité de numismatique celtique -IMéthodologie des ensembles, Paris, Les Belles Lettres, 1973, p. 366.

21. Posé dès 1995 (Mangin el al., 1995), ce problème est assurément lourd d'implications historiques fondamentales pour l'est des Gaules. 
ne commencer qu'au $\mathrm{I}^{\mathrm{er}} \mathrm{s}$. de notre ère. La question de la continuité ou de la discontinuité entre l'âge du Fer et l'époque romaine dans les zones de production primaire reste ouverte.

En revanche, le système d'organisation de la production primaire (extraction minière et réduction) mis en place à l'époque romaine apparaît maintenant assez clairement dans l'est de la Gaule. Deux niveaux ont été mis en évidence : d'une part un assez vaste espace, un « district » couvrant plusieurs centaines de kilomètres carrés, où la sidérurgie semble être l'activité dominante sinon principale; d'autre part, des zones limitées dépendant peut-être de centres urbains d'importance diverse. L'activité secondaire de transformation apparaît, elle, disséminée en milieu urbain ou rural, avec, pour certains secteurs géographiques, différents niveaux d'organisation qui coexistent.

\section{LE MORVAN DU NORD, UN GRAND DISTRICT PUBLIC DE PRODUCTION PRIMAIRE}

Les ateliers de réduction - tels qu'ils peuvent apparaître en prospection dans les forêts et les prairies à travers les dépôts de scories - sont répartis sur une surface de $400 \mathrm{~km}^{2}$, nettement séparée des vastes zones productives de la Nièvre ou de l'Yonne. Il s'agit donc d'un « district » géographique clairement délimité (Mangin et $a l ., 1992)$.

La répartition des sites de travail se présente sous trois formes correspondant à trois types de régions naturelles.

Au nord-est, sur un môle cristallin recouvert de sédiments triasiques, le plateau de Thoste, sont concentrés des dizaines d'ateliers utilisant le minerai en roche de l'Hettangien ou son altération et, secondairement, du minerai en grains d'origine pédogénétique. Des installations fixes existent, par exemple des habitats liés aux ateliers et un « village ».

Sur les plateaux et dans les vallées du Morvan intérieur cristallin sont dispersés des dizaines d'ateliers exploitant le même minerai des lambeaux sédimentaires, conservés ici ou là, ou du minerai pédogénétique. Aucun habitat permanent n'a pu être décelé dans cet espace.

Enfin, au nord et à l'est, sur les plateaux de la périphérie de l'Auxois, le minerai des limons superficiels est exploité par des ateliers dépendant spatialement de grosses villae.
Les 200 ateliers répertoriés relèvent tous de la réduction, sauf deux forges de village ou de villa. Leur implantation est indépendante de la topographie et de l'hydrographie, même si les bas de versants sont privilégiés ; elle s'explique d'abord par la présence de minerai et aussi, évidemment, par la proximité du combustible.

Tout cet ensemble se situe au nord de la bourgade de Saulieu. À l'inverse, au sud, fermes et villae sont nombreuses. Ce ne sont pas les conditions naturelles pour l'exploitation rurale - identiques - qui expliquent cette opposition, mais des raisons humaines : le Nord productif apparaît comme une zone non aedificandi dont il est tentant d'attribuer la création à la cité éduenne dans laquelle se situe cet espace (à moins que l'on veuille en faire une annexe du grand district d'État postulé de l'Yonne), tandis que le Sud dépourvu de minerai est laissé à l'exploitation rurale privée ${ }^{22}$. Saulieu apparaitrait ainsi comme le probable centre de gestion et d'échanges de la zone, surtout à en juger par l'étoile de routes qui en rayonne. On peut supposer que la production sidérurgique des villae périphériques est libre, elle aussi, évidemment contre paiement d'un vectigal, c'est-à-dire d'une taxe dont on peut s'acquitter en livrant du fer, comme c'est le cas au Moyen Âge dans le Jämtland suédois ${ }^{23}$.

La présence au nord-est de Saulieu d'un village de mineurs (Montlay-en-Auxois) d'un type original renforce ces hypothèses: le long d'un chemin qui conduit à Saulieu, à proximité d'un sanctuaire des eaux de type indigène et parmi de nombreux ferriers, une douzaine de maisons sont alignées selon une organisation stricte qui manifeste une mise en place volontaire par une autorité publique.

Volonté de la cité éduenne? C'est aussi la question des débouchés de la production qui se pose : Autun est à $24 \mathrm{~km}$; nombre d'ateliers de forge y sont attestés sous le Haut-Empire et deux fabricae d'armes y fonctionneront au Bas-Empire. Ainsi, le schéma qui se dégage est celui d'une zone minière où la mise en valeur s'effectue, d'une part, en vertu d'une adaptation à la localisation des ressources minérales et, d'autre part, en fonction d'un partage de l'espace entre deux modes de gestion : privée en périphérie et publique dans le cœur de la zone avec des habitats aux marges, d'où mineurs et ferrons peuvent

22. Argumentation appuyant l'hypothèse d'un district public dans Mangin et al., 1992, p. 225-237.

23. Cf. note 9, p. 13 : G. Magnusson, op. cit., 1986, p. 311-312. 
aller travailler quotidiennement ou saisonnièrement dans un rayon de 10 à $15 \mathrm{~km}$.

\section{UNE PRODUCTION PRIMAIRE DE NIVEAU INTERMÉDIAIRE OU LOCAL PLUS DISPERSÉE}

En dehors du Morvan, si les sites ayant livré des scories de réduction sont nombreux, ils n'appartiennent le plus souvent, quand il sont datés, qu'à l'époque postérieure ; de plus, ils sont, la plupart du temps, disséminés sur l'ensemble du territoire; néanmoins deux modestes concentrations sont reconnues.

En Franche-Comté, où n'existent pas de grands ateliers de réduction, on a mis en évidence, une zone de petite production (dite zone de Berthelange-forêt d'Arne avec une extension dans la région de Byans sur une voie importante), à proximité de la capitale des Séquanes, Vesontio/Besançon. Plus de 60 sites à scories ont été repérés sur une surface d'environ $100 \mathrm{~km}^{2}$. Ils sont liés à des habitats dont la modestie contraste avec l'importance des villae des zones voisines qui sont établies sur des terres riches. Il s'agit toujours de petits ateliers voués à la réduction du minerai local sur lequel ils sont implantćs (Laurcnt et al., 1994; Laurcnt, 1997) ${ }^{24}$. Lcs travaux actuels cherchent à dater ces sites et à préciser le fonctionnement socio-économique de ce petit district en tentant de répondre à quatre questions :

- de quelle manière les ateliers étaient-ils en relation avec les villae situées à proximité ?

- ce groupement homogène de petits habitats et d'ateliers dépendait-il d'une autorité organisatrice extérieure?

- est-ce la présence de ressources minières attestées qui explique la mise en place de cette zone sidérurgique ou la proximité du chef-lieu de cité ?

- enfin, où était transformée sa production, puisque très peu de forges semblent avoir existé dans les villae voisines?

Tout se passe comme si cette petite production n'était qu'une activité de substitution temporaire ou une activité complémentaire de l'agriculture, comme ce fut le cas pour les fermes du Jämtland suédois au Moyen Âge ${ }^{25}$.

24. Gisements de minerai de fer pisolithique plio-pléistocène, centrés sur la forêt d'Arne.

25. (f. note 9, p. 13 : G. Magnusson, op. cil., 1986.
Dans le Mâconnais, plusieurs campagnes de prospection systématique ont confirmé l'existence d'un district comparable, bien délimité, couvrant une superficie de $150 \mathrm{~km}^{2}$ et renfermant plus de 45 sites de réduction (Boukezzoula, 1995). La partie nord est la plus riche en vestiges, particulièrement autour du hameau de Blany (commune de Laizé). La majorité des ateliers est liée à des habitats gallo-romains (villae et petite agglomération), implantés à proximité immédiate des voies de communication terrestre (voie d'Agrippa) ou fluviale (la Saône). Les sites se distribuent pour la plupart sur un même axe nordsud, principalement sur la rive droite de la Saône. La fouille d'un atelier a livré deux bas fourneaux et l'étude archéométrique des scories a confirmé l'utilisation du minerai de fer des minières toutes proches de Blany, dont les vestiges d'exploitation sont conservés. Cette zone de production, un " district " modeste mais bien caractérisé, pourrait être en relation avec le travail métallurgique attesté dans la ville antique de Mâcon, des origines celtiques de cet oppidum jusqu'à l'Empire tardif où, comme pour Autun, une fabrica est attestée par la Notitia Dignitatum.

Ailleurs comme en Lorraine ou dans le reste de la Franche-Comté, les ateliers de réduction isolés d'époque romaine sont rares : ils sont parfois liés directement aux agglomćrations; il cn cxistc aussi dans les campagnes au sein d'un nombre non négligeable de villae, les ateliers jouxtant les bâtiments.

En ville, y compris dans les capitales, l'activité de réduction est probablement seulement conjoncturelle, par exemple à Autun lors de la phase de construction de la ville. On la trouve aux portes de quelques agglomérations secondaires (10 cas sur 77 en Bourgogne/FrancheComté) ; elle peut exister ponctuellement dans les ateliers de forge : la question reste posée pour certains ateliers d'Alésia (Mangin et al., 2000a) ${ }^{26}$. Toutefois, c'est

26. Les opinions ont beaucoup varié sur cette question : on a d'abord vu partout de la réduction quand on trouvait un foyer avec scories sur un oppidum ou en ville ou villa; puis la réduction a été niée partout hors des districts de production du type du Morvan. On peut aujourd'hui dresser un bilan plus circonstancié qui montre que la situation est moins tranchée et doit tenir compte de cas très divers : des ateliers des villages de mineurs jusqu'aux bas fourneaux temporaires dans les faubourgs de capitales ou permanents dans ceux de bourgades spécialisées, of. le bilan fourni pour la Bourgogne et la Franche-Comté dans J.-P. Petit, M. Mangin dir., Les agglomérations secondaires : la Gaule Belgique, les Germanies et l'Occident romain, Actes du colloque de BliesbruckReinheim/Bitche, oct. 1992, Paris, éd. Errance, 1994, p. 49-51 et J.P. Petit, M. Mangin dir., Atlas des agglomérations secondaires de la Gaule Belgique et des Germanies, Paris, éd. Errance, 1994, 293 p. 
majoritairement la forge qui caractérise l'activité sidérurgique dans les villes, les villae et les fermes.

\section{LE TRAVAIL DE FORGE DANS LES HABITATS}

Le travail de postréduction au sens large est identifié sur un grand nombre de sites de l'aire d'étude, mais peu de cas sont connus avec une précision suffisante. Les ateliers urbains sont souvent les mieux documentés et sont représentés dans une large majorité d'agglomérations. Dans les zones rurales où des recensements systématiques ont pu être réalisés, comme le Finage dolois ou le Haut-Auxois, la proportion des habitats pourvus d'une forge est d'environ un tiers.

\section{LE TRAVAIL DE FORGE DANS LES AGGLOMÉRATIONS}

La forge est le type d'atelier de loin le plus répandu en ville : $40 \%$ des cas en Bourgogne/Franche-Comté par exemple. Les déchets sont partout, y compris dans les capitales de cités : à Avenches (en Suisse), ils sont dispersés sur une vingtaine d'emplacements (Serneels, 1993, p. 162); à Autun (Bourgogne) sur treize; à Metz (Lorraine), dans au moins quatre endroits. Il semble pourtant exister des quartiers privilégiés : à Autun, dans le grand quartier de bronziers qui vient d'être récemment fouillé, les 5 forges sont d'importance secondaire par rapport aux 50 ateliers de bronziers au sein desquels elles sont dispersées. Il en est de même dans l'Alésia gauloise et augustéenne (petits ateliers de forgerons : lots de $12 \mathrm{~m}$, entre grands ateliers de bronziers : lots de $20 \mathrm{~m}$ ) le long du " proto-forum ». D'une manière générale, les ateliers de forgerons sont souvent associés aux ateliers de bronziers (Alésia, Bliesbruck, Metz), parfois à une autre activité artisanale comme la céramique (MathayMandeure, peut-être Florange). Il peut donc s'agir de petits ateliers à vocation locale au service des artisanats principaux. Mais dans plusieurs cas, il s'agit sans doute d'une activité importante, même si elle n'est pas exclusive d'autres fonctions (Alésia, Bliesbruck, Entrains).

\section{LE TRAVAIL DE FORGE DANS LES HABITATS RURAUX}

Nombre de villae et de fermes sont dotées d'une forge. Plus d'une centaine sont répertoriées, mais peu sont étudiées. La densité de forges rurales est très inégale selon les régions. Dans deux zones bien étudiées (le Finage dolois : 30 forges ; le Haut-Auxois : 45), le pourcentage d'habitats ruraux pourvus d'une forge (environ $30 \%$ ) semble correspondre à une réalité antique et non à l'état des recherches. Il existe donc des régions rurales où la fabrication de l'équipement en fer était systématique. Ainsi, à la spécialisation d'agglomérations artisanales fabriquant l'outillage des autres artisans, correspondrait la fabrication de l'outillage agricole au sein des villae ou des petites agglomérations rurales.

À Saint- $\Lambda$ ubin, au cœur du Finage dolois (Jura, Franche-Comté), le nombre et la dimension des culots de forge sont sans rapport avec ceux des petites forges de la plupart des fermes et villae de la région. Les analyses chimiques effectuées tendent à confirmer ce constat : les scories de trois sites correspondraient à des déchets d'épuration du fer brut (Saint-Aubin, agglomération centrale du Finage, Molay, grande villa et la grande forge de Choisey, sur la voie Besançon-Chalon). Les scories de tous les ateliers liés aux fermes du Finage sont des déchets de forge classiques (Raissouni, 1995). Sur ce territoire, les recherches archéométriques permettent donc de proposer un modèle d'organisation et de diffusion de la production avec, d'une part, des sites peut-être spécialisés dans la transformation des produits bruts en produits forgeables (forges d'agglomération, de grosse villa et de site routier) et, d'autre part, de simples forges de fermes où se fabriquaient uniquement les objets utilisés sur place.

Or, ce modèle, avancé avec prudence dans la conclusion de la thèse de B. Raïssouni, se retrouve - argumenté plus largement et élaboré d'une façon totalement indépendante - dans l'étude parallèle de la région du HautAuxois autour d'Alésia (Mangin et al., 2000b). L'étude archéologique et archéométrique des 45 ateliers répertoriés autour d'Alésia fait, en effet, ressortir deux faits majeurs.

D'une part, leur liaison préférentielle avec les activités liées au trafic plutôt qu'avec la terre: ce ne sont pas en majorité des ateliers ruraux au sens strict; dans trois cas sur quatre, ils apparaissent comme des forges routières. Ce constat est à comparer avec celui du Finage, où les sites implantés sur les voies - les forges du Mazeret à Saint-Aubin et l'atelier routier de Choisey - sont les plus importants.

D'autre part, technologiquement, ils se distribuent en deux catégories : les ateliers des trois agglomérations de la région (la ville d'Alésia, le village de Blessey-Salmaise et la station routière de Sombernon) ne pratiquent pas seu- 
lement le simple forgeage d'objets, mais assument aussi l'épuration de fer brut importé d'une région extérieure (le Châtillonnais peut-être), tandis que l'ensemble des autres ateliers des sites ruraux isolés ou des sites installés sur les voies sont de simples forges d'élaboration d'objets (à l'exception d'un cas d'atelier situé sur un carrefour).

Il a fallu une étude fine associant terrain et laboratoire, archéologie et archéométrie (analyses chimiques et examens métallographiques approfondis conduits d'une façon totalement intégrée, cf. supra, p. 12-13), pour aboutir à ce type de résultat, qui était totalement inattendu au départ. C'est dire l'intérêt qu'il y aurait à disposer d'un certain nombre d'études régionales de ce type pour voir si Finage et Haut-Auxois sont des exceptions dans les Gaules ou si ces microrégions entrent dans une catégorie nouvelle, celle de « régions de forge », spécialisées dans les étapes de transformation métallurgique (Faivre et al., 1998).

\section{LA FIN DE L'ANTIQUITÉ ET LE HAUT MOYEN ÂGE : IAA MODIFICATION DU CADRE DE LA PRODUCTION}

Sous le Haut-Empire, seules quelques zones de production primaire spécialisées semblent donc approvisionner de vastes espaces de consommation. Cette organisation de la production sidérurgique change au cours du Bas-Empire. Mais, faute de données suffisamment précises, la manière dont s'effectuent ces modifications entre le III' et le $\mathrm{V}^{\mathrm{c}} \mathrm{s}$. n'est pas bien connue. La fouille de quelques ateliers de l'Antiquité tardive et du haut Moyen Âge, et, parmi les sites découverts au cours des programmes d'inventaire, un nombre important de sites datés de cette époque permettent de saisir des modifications aussi bien sur le plan des techniques que sur celui de l'organisation de la production.

\section{DES CHANGEMENTS TECHNIQUES}

Sur le gros atelier de réduction des Clérimois (Yonne), pendant tout le Haut-Empire, ce sont les techniques de production attestées dès le II ${ }^{\mathrm{s}} \mathrm{s}$. avant notre ère (grands ateliers fonctionnant par batteries de deux bas fourneaux du type Burgenland) qui continuent d'être utilisées (Dunikowski, Cabboï, 1995). Le passage des Sénons sous l'autorité romaine n'a donc pas entraîné de changement technique : apparemment, Rome s'intéressait aux revenus tirés de la production, non à la manière de l'obtenir.

À l'inverse, le changement qui intervient dès le $I^{c} \mathrm{~s}$. est clairement d'ordre technique. La production ne s'effectue plus dans des grands fourneaux disposés en batterie, mais dans des bas fourneaux indépendants, d'un type nouveau que l'on retrouve au début du haut Moyen Âge en Suisse (Pelet, 1973, 1993 ; Eschenlohr, Serneels, 1991), en Lorraine (Leroy, 1997) et en Franche-Comté (Laurent et al., 1994) ${ }^{27}$. Cette innovation technologique correspond-elle à un changement social ? La question mérite au moins d'être posée : les bas fourneaux du haut Moyen Âge sont-ils des importations "barbares " ? Si c'est le cas, la date haute de leur introduction dans l'est de la France peut-elle être mise en rapport avec la "barbarisation" de cette région qui commence très tôt avec l'introduction de «supplétifs » barbares dans les armées des frontières? (Rappelons à ce propos les réflexions présentées par le "groupe de l'Est " au symposium de Norberg, en Suède en mai 1995: Mangin et al., 1995).

\section{UNE ORGANISATION DE LA PRODUCTION PRIMAIRE DE TAILLE PLUS MODESTE?}

À partir du Ve et certainement aux $\mathrm{VI}^{\mathrm{e}}$ et $\mathrm{VII}^{\mathrm{e}} \mathrm{s}$., l'organisation de la production s'est modifiée. L'industrie est nettement plus disséminée et se développe à petite échelle dans de nombreuses régions. Les ateliers sont de dimensions moindres, avec un, deux ou trois fourneaux et un tas de déchets ne dépassant pas quelques dizaines de mètres cubes : Frouard et Ludres en Lorraine, Ferrières-lès-Bois et Louvatange en FrancheComté. La production du fer semble être devenue une industrie de proximité avec de nombreux petits centres satisfaisant la demande locale ${ }^{28}$. On constate la

27. La similitude morphologique des modèles de bas fourneaux découverts dans l'est de la Gaule (en Bourgogne du nord, I corraine centrale, Franche-Comté et Suisse occidentale) est tout à fait frappante et révélatrice d'une indéniable unité technologique, et ce malgré des différences notables dans les types de minerais utilisés.

28. Cette multiplication des ateliers est bien illustrée en Lorraine où les premiers véritables districts sidérurgiques se constituent entre le VII ${ }^{*}$ et le $\mathrm{X}^{\mathrm{e}} \mathrm{s}$., au cœur des vallées encaissées du Plateau de Haye et du PaysHaut (région des Côtes de Moscllc) ; ccrtains forricrs sont ccpendant de très gros volume (Ludres-village, Avril, Moyeuvre-Grande). 
même situation de l'autre côté du Jura, sur le versant suisse et tout récemment au coude du Rhin au-dessus de Martigny où la quinzaine de petits ferriers du Mont Chemin est datée des années 550-650 (Beck, Serneels, 1998).

Les ateliers de réduction paraissent assez systématiquement isolés, distants des habitats. Dans les villages, on trouve seulement les vestiges du travail du métal (forge au sens large). L'utilisation du fer semble être plus restreinte (armement, outillage, habillement).

L'interruption des grands courants de circulation en place pendant la Pax romana explique peut-être cet éclatement. En Lorraine et en Franche-Comté comme en Suisse occidentale, il ne fait qu'accentuer la situation antérieure : il n'y a pas de rupture aux IV et $V^{e} s$., mais continuité dans la mesure où il s'agissait déjà là de petite métallurgie dispersée. Il en va différemment, semble-t-il, en Bourgogne, espace où, dans les cités existait une grosse production; mais si rupture il y a, elle reste à confirmer par une documentation plus précise. C'est ainsi que chez les Éduens, on ne connaît pas de grands sites de réduction datés du Bas-Empire ou du haut Moyen Âge ; même si, parmi les ferriers du Morvan du nord ou du Mâconnais, il en existe de cette époque comme c'est probable mais pas encore attesté, ils ne correspondent pas à une production importante; or celle-ci devait pourtant exister ne serait-ce que pour l'approvisionnement des fabricae d'Autun et de Mâcon. Chez les Sénons, les ateliers des Clérimois fonctionnent seulement jusqu'au $\mathrm{V}^{\mathrm{e}} \mathrm{s}$., mais cet exemple clairement établi est-il représentatif, la chronologie des ferriers de cette vaste zone productive restant à établir?

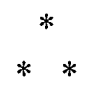

\section{PEUPLES DE L'EST DE LA GAULE ET SIDÉRURGIE}

Les inventaires qui viennent d'être dressés pour les ateliers sidérurgiques et les premières synthèses parues ou à paraitre des auteurs de cette communication et d'autres chercheurs travaillant dans l'est de la France révèlent clairement l'opposition entre deux groupes de peuples gaulois, sensible dès la fin de la Protohistoire et éclatante à l'époque romaine.
D'une part, deux espaces géographiques où domine l'activité sidérurgique, ceux qu'occupent les Sénons et les Éduens, et pour une part les Lingons-Mandubiens.

D'autre part, des régions où la métallurgie du fer est présente partout mais sous la forme de petites unités dispersées ou groupées dans des zones d'importance modeste, comme chez les Séquanes, les Leuques et les Médiomatriques.

Cette prééminence de la cité éduenne, à côté de celle des Sénons, explique probablement qu'elle dispose au Bas-Empire, à elle seule, de trois des neuf fabricae d'armes des Gaules, deux dans sa capitale, Autun (Augustodunensis loricaria, balistaria et clibanaria, Augustodunensis scutaria), la troisième dans un de ses ports sur la Saône, Mâcon (Matisconensis sagittaria) (Notitia Dignitatum, 9, 32-34) ${ }^{29}$.

La situation change avec la fin de l'Empire romain. La production séquane semble se multiplier (y compris dans la partie orientale, l'Helvétie, englobée dans la Maxima Sequanorum), celle des Leuques et des Médiomatriques aussi, alors qu'il faut attendre le Moyen Âge classique (XII -XIV'e s.) pour que la métallurgie reprenne, dans l'actuelle Bourgogne (Éduens, Sénons et partiellement Lingons) ou dans l'ensemble de la Lorraine, une importance qu'elle conservera jusqu'au XX's. Ce changement concerne les peuples les plus proches du limes rhénan: est-ce un hasard?

Bien d'autres questions restent à élucider. Ces remarques permises par les acquis récents des recherches sont, pour une part, plus des interrogations que des affirmations. Il ne sera possible de les considérer comme de véritables hypothèses de travail que lorsque nous disposerons de dizaines d'ateliers fouillés et datés précisément. On en est encore loin. Mais une comparaison de la situation des connaissances dans l'est de la France entre 1983 et 1998 (un millier d'ateliers prospectés et plus d'une cinquantaine de bas fourneaux fouillés sur plus d'une dizaine d'ateliers en une décennie et demie) montre que les choses progressent vite. Les programmes en cours, appuyés sur ces acquis de terrain et de laboratoire, doivent donc afficher cet objectif. Il comporte aussi évidemment la recherche systématique des habitats des travailleurs : on ne les trouvera que si on les cherche dans les campagnes comme on l'a fait quelque peu dans les villes et les bourgades.

29. Notitia Dignitatum, éd. O. Seek 1876, rééd. Minerva, Francfort-surle-Main, 1962, p. 145-146. 